\title{
The Therapy of Cancer
}

\author{
Jiayuan Jiao, Yi Bi and Baoshan Chai* \\ Pharmaceutical Research Laboratory, Shenyang Research Institute of Chemical Industry Company Ltd., Shenyang, 110021, China
}

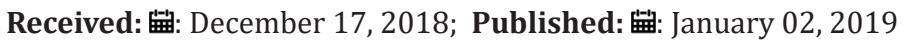

*Corresponding author: Baoshan Chai, Pharmaceutical Research Laboratory, China

\begin{abstract}
Cancer is becoming one of the major diseases with serious hazards to human health. To find efficient treatment protocols has always been the important subject in cancer studies. According to the statistical data of the world health organization, the incidence and mortality of cancer show a continuous increasing trend. Therefore, cancer treatment is widely concerned. Based on clinical knowledge. The therapy of cancer has been proved to be with significant efficacy and superiorities in both experimental and clinical trials. The principle of therapy of cancer and some recent technologies in cancer therapy were summarized.
\end{abstract}

Keywords: Cancer; Therapy

\section{Mini Review}

With the extension of the average life expectancy of human beings, the threat of cancer to human beings has become increasingly prominent and it has become the first cause of death for urban and rural residents in China. At present, there are many kinds of malignant tumors, different types of properties, different tissues and organs involved, different disease periods, and different responses to various treatments, so most patients need comprehensive treatment. The so-called comprehensive treatment is based on the patient's physical condition, the pathological type of the tumor, the scope of invasion, etc., comprehensively using surgery, chemotherapy, radiotherapy, immunotherapy, traditional Chinese medicine treatment, interventional therapy, microwave therapy, etc., in order to greatly improve the cure and improve the quality of life of patients.

\section{Surgical Treatment}

In theory, if the tumor cells are completely removed by surgery, the cancer can be cured. Surgical resection is still the treatment of choice for early or earlier solid tumors. According to the purpose of the surgery, it can be divided into the following categories:

Radical Surgery [1]: Because the malignant tumor grows fast, there is no envelope on the surface, it has no obvious boundary with the surrounding normal tissue, local infiltration is obvious, and it can be transferred through lymphatic vessels. Therefore, the surgery should completely remove the tumor and a range of normal tissues around it and the lymph nodes that may be violated. This kind of surgery is suitable for patients with limited tumor range, no distant metastasis, and good physical condition. According to the scope of resection, it is divided into tumor resection, extensive resection, radical operation and extended radical surgery.

Palliative Surgery: It has a wide range of tumors. Patients with advanced metastases who cannot be treated for radical surgery can only remove some tumors or perform some symptoms-reducing operations, in order to relieve pain, maintain nutrition and prolong life. Surgery, digestive tract short circuit and other operations. Advanced tumors can do most of the tumor resection, reduce the tumor burden, and lay the foundation for future radiotherapy, chemotherapy or other treatment. For example, advanced ovarian cancer can palliatively remove most ovarian tumors [2], and undergo secondary surgery after chemotherapy to remove residual lesions, which can significantly prolong the survival of patients.

Diagnostic Surgery: Specimens for pathological examination [3] of the tumor obtained by different surgical methods, such as puncture or incision of small pieces of tissue during surgery. For deep visceral masses, it is necessary to open the chest, open the laparotomy or craniotomy. After the intraoperative pathological examination is confirmed, the therapeutic operation is performed immediately.

Prophylactic Surgery [4]: For precancerous lesions to prevent malignant transformation or progression to advanced cancer, such as familial colonic polyposis, can benefit from prophylactic 
colectomy, because such patients do not remove the colon, about half of them can develop colon cancer after the age of 40 , and almost $100 \%$ develop colon cancer after the age of 70 .

Surgery of Metastases [5]: For a single lung, liver, bone and other metastases, resection can still achieve a 5-year survival rate. For example, primary lung cancer has only a single metastatic lesion and the primary lung cancer can be completely resected [6]. The primary tumor and metastasis can be performed simultaneously or sequentially.

\section{Chemotherapy}

It treats cancer with drugs that kill cancer cells [7]. Since the biggest difference between cancer cells and normal cells is rapid cell division and growth, the principle of anticancer drugs is usually to inhibit the growth of cancer cells by interfering with cell division, such as inhibiting DNA replication or preventing chromosome segregation. Most chemotherapy drugs are not specific, so they kill normal tissue cells that undergo cell division, and often damage healthy tissues that need to be split to maintain normal function, such as intestinal mucosal cells. However, these tissues usually repair themselves after chemotherapy. Because some drugs can be combined for better results, chemotherapy often uses two or more drugs at the same time, called "comprehensive chemotherapy," and most patients use chemotherapy in this way.

\section{Radiation Therapy}

It uses radiation to kill cancer cells and shrink tumors. Radiation therapy can be via in vitro radiation therapy or in vivo proximity radiation therapy [8]. Since cancer cells grow and divide faster than normal cells, the genetic material of the cells is destroyed by radiation, which prevents cells from growing or dividing, thereby controlling the growth of cancer cells. However, the effects of radiation therapy can only be limited to areas that receive exposure. The goal of radiation therapy is to destroy as many cancer cells as possible while minimizing the impact on neighboring healthy tissue. Although radiation exposure can cause damage to both cancer cells and normal cells, most normal cells can recover from radiation-induced damage.

\section{Targeted Therapy}

Targeted therapies [9] have seen significant effects in the treatment of certain types of cancer since the late 1990s, and they are as effective in treating cancer as chemotherapy, but with fewer side effects than chemotherapy. It is also a very active research area at the moment. The principle of this treatment is the use of small molecules that specifically target abnormal or dysregulated proteins of cancer cells, such as tyrosine kinase inhibitors, for the treatment of EGFR (epidermal growth factor receptor) sensitive mutations in non-small cell lung cancer. The curative effect is remarkable, but the emergence of drug resistance genes is currently the main obstacle hindering further improvement of curative effect.

\section{Immunotherapy}

Immunotherapy uses the immune mechanism in the body to fight against tumor cells [10]. There have been many immunotherapies against cancer in research. Currently, cancer vaccine therapy and monoclonal antibody therapy are more advanced, immune cell therapy is the latest development of treatment technology in recent years.

\section{Chinese Medicine Treatment}

Combined with surgery, radiotherapy and chemotherapy can reduce the side effects of radiotherapy and chemotherapy, promote patient recovery and enhance the tolerance to radiotherapy and chemotherapy [11].

\section{Gene Therapy}

By introducing foreign genes into the human body to correct gene defects, the concept of gene therapy [12] for malignant tumors has expanded from correcting gene defects to introducing foreign genes into the human body, eventually achieving direct or indirect inhibition or killing of tumor cells.

\section{Endocrine Therapy}

The occurrence and growth of certain tumors are closely related to hormones in the body, so they can be treated by changing the endocrine status. For example, sex hormones can be used for palliative treatment of breast cancer [13], prostate cancer, and endometrial cancer.

\section{Laser Treatment}

The advantages of high density, good parallelism, and accurate positioning of the laser are used to make a bloodless resection of the lesion after moderate focus [14].

\section{Conclusion}

Although there are many treatments for cancer, chemotherapy is still the main treatment in clinical. However, as technology continues to advance, the development of cancer treatment drugs will be dominated by targeted drugs plus immunotherapy. The field worthy of further to study. To provide new ideas for the best personalized treatment for different patients. And new methods play an important role in the fight against cancer.

\section{References}

1. Wyld L, Audisio RA, Poston GJ (2015) The evolution of cancer surgery and future perspectives. Nature Reviews Clinical Oncology 12(2): 115124.

2. Miller D, Nevadunsky N (2018) Palliative Care and Symptom Management for Women with Advanced Ovarian Cancer. Hematologyoncology clinics of north america 32(6): 1087-1102.

3. Bejarano PA, Berho M (2015) Examination of Surgical Specimens of the Esophagus. Archives of pathology \& laboratory medicine 139(11): 14461154.

4. Ludwig KK, Neuner J, Butler A, Geurts JL, Kong AL (2016) Risk reduction and survival benefit of prophylactic surgery in BRCA mutation carriers, a systematic review. American journal of surgery 212(4): 660-669.

5. Akgül Ö, Çetinkaya E, Ersöz Ş, Tez M (2014) Role of surgery in colorectal cancer liver metastases. World journal of gastroenterology 20(20): 6113-6122.

6. Sun Z, Sui X, Yang F, Wang J (2018) Effects of primary tumor resection on the survival of patients with stage IV extrathoracic metastatic non-small cell lung cancer: A population-based study. Lung Cancer pii: S01695002(18): 306390-306391. 
7. Hartner L (2018) Chemotherapy for oral cancer. Dental clinics of North America 62(1): 87-97.

8. Badiyan SN, Molitoris JK, Chuong MD, Regine WF, Kaiser A (2017) The Role of radiation therapy for pancreatic cancer in the adjuvant and neoadjuvant settings. Surgical Oncology Clinics 26(3): 431-453.

9. Tsimberidou AM (2015) Targeted therapy in cancer. cancer chemotherapy and pharmacology 76(6): 1113-1132.

10. Yang Y (2015) Cancer immunotherapy: harnessing the immune system to battle cancer. The Journal of clinical investigation 125(9): 3335-3337.

11. Wu Y, Wang D, Yang X, Fu C, Zou L, et al. (2019) Traditional Chinese medicine Gegen Qinlian decoction ameliorates irinotecan chemotherapy-

ISSN: 2574-1241

DOI: $10.26717 / B J S T R .2019 .12 .002268$

Baoshan Chai. Biomed J Sci \& Tech Res

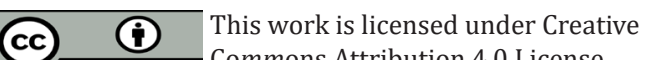

Submission Link: https://biomedres.us/submit-manuscript.php induced gut toxicity in mice. Biomedicine pharmacotherapy 109: 22522261.

12. Husain SR, Han J, Au P, Shannon K, Puri RK (2015) Gene therapy for cancer: regulatory considerations for approval. Cancer gene therapy 22(12): 554-563.

13. Reinbolt RE, Mangini N, Hill JL, Levine LB, Dempsey JL, et al. (2015) Endocrine therapy in breast cancer: the neoadjuvant, adjuvant, and metastatic approach. Seminars in oncology nursing 31(2): 146-155.

14. Mirza FN, Khatri KA (2017) The use of lasers in the treatment of skin cancer: A review. Journal of Cosmetic and Laser Therapy 19(8): 451-458.

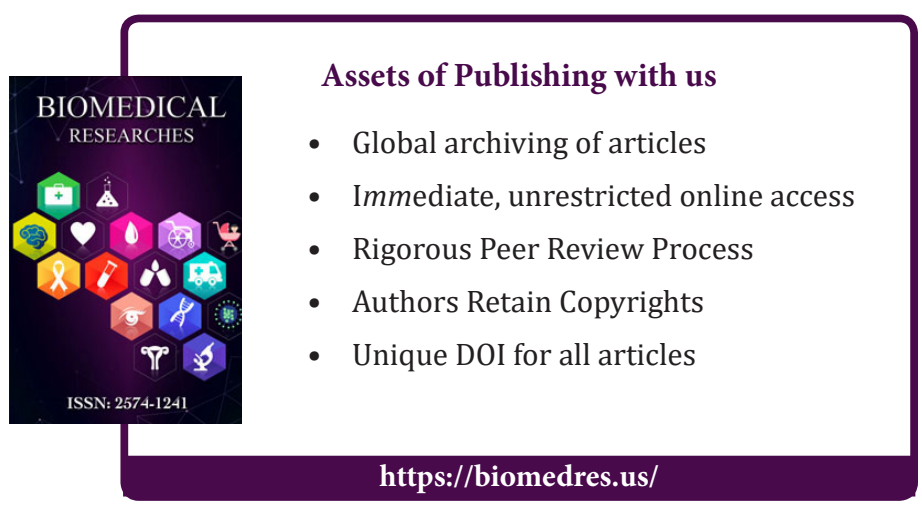

